\title{
Notes
}

\begin{abstract}
Hisao Tsukamoto, Hidetoshi Yoshimura, and Satoshi Toki : Metabolism of Drugs. XVIII.) The Metabolic Fate of Methylhexabital (5-Cyclohexenyl3,5-dimethylbarbituric Acid). (8). ${ }^{1)}$ The Quantitative Determination of Main Biotransformation Products of Methylhexabital in the Urine of Rabbits by Ultraviolet Spectrophotometry.
\end{abstract}

(Pharmaceutical Institute, Medical Faculty, University of Kyushu*)

The latest work $\mathrm{x}^{1)}$ in this series has shown that ureide [1-(2-cyclohexenylpropionyl: -3-methylurea)], 3-OH-MHB** (5-(3-hydroxy-1-cyclohexeny1)-3,5-dimethylbarbituricac: id), 3-keto-MHB [5-(3-oxo-1-cyclohexeny1)-3,5-dimethylbarbituric acid] and 3-keto-norMHB $[5$-(3-oxo-1-cyclohexenyl)-5-methylbarbituric acid] were separated and identified: by buffered paper chromatography from the urine of rabbits administered methylhexabital (MHB, 5-cyclohexenyl-3,5-dimethylbarbituric acid).

A method of estimating a small amount of urinary metabolites of MHB was required during the course of an investigation on the metabolism of MHB.

This paper describes a method for the simultaneous determination of three main. metabolites (3-OH-MHB, 3-keto-MHB, and 3-keto-nor-MHB) in rabbit urine by the use: of buffered paper chromatography in conjunction with ultraviolet spectrophotometry, and presents a relative concentration of each metabolite in the 24-hour urine.

This procedure was essentially the same as those described in the previous paper ${ }^{2)}$. about the excretion rate of metabolites of ethylhexabital (5-cyclohexenyl-5-ethylbarbituric acid). The estimation of the concentration of ureide and MHB-M (VI) was not made, because the former had no absorption peak and structure of the latter was not. clarified as yet.

\section{Materials and Methods***}

MHB (m.p. $142 \sim 143^{\circ}$ ) was supplied by Dainippon Seiyaku Co. Ltd., 3-keto-MHB (m.p. $160 \sim 161^{\circ}$ ) and 3-keto-nor-MHB (m.p. 215 216 (decomp.)) were prepared by the oxidation $^{3)}$ of $\mathrm{MHB}$ and nor-MHB, respectively. 3-OH-MHB was obtained ${ }^{4,5)}$ from the urine of rabbits. The extinction measurements were made with a Hitachi photoelectric spectrophotometer with standard $10-\mathrm{mm}^{2}$ "quartz absorption cell.

In borate- $\mathrm{NaOH}$ buffer ( $\mathrm{pH}$ 11) solution, the extinction at 238,240 , and $244 \mathrm{~m} \mu$ was directly propotional to the concentration of 3-keto-nor-MHB, 3-keto-MHB, or 3-OHMHB, in accordance with the Lambert-Beer law (Fig. 1).

* Katakasu, Fukuoka (塚元久雄, 吉村实敏, 上岐 㖞).

* As shown in previous study 3-OH-MHB is composed from two diastereoisomeric $\alpha-$ and $\beta-3-$ $\mathrm{OH}-\mathrm{MHB}$, and both exhibit the same $\mathrm{Rf}$ value and identical ultraviolet absorption spectra.

*** All mèlting points are uncorrected.

1) Part XVII : H. Tsukamoto, H. Yoshimura, S. Toki : This Bulletin, 6, 15(1958).

2) H. Tsukamoto, E. Takabatake, T. Ariyoshi : Ibid, 3, 459(1955).

3) H. Tsukamoto, H. Yoshimura, S. Toki : Ibid., 4, 363(1956)

4) Idem.: Ibid., 4, 368(1956).

5) H. Yoshimura : Ibid., 5, 561(1957) 


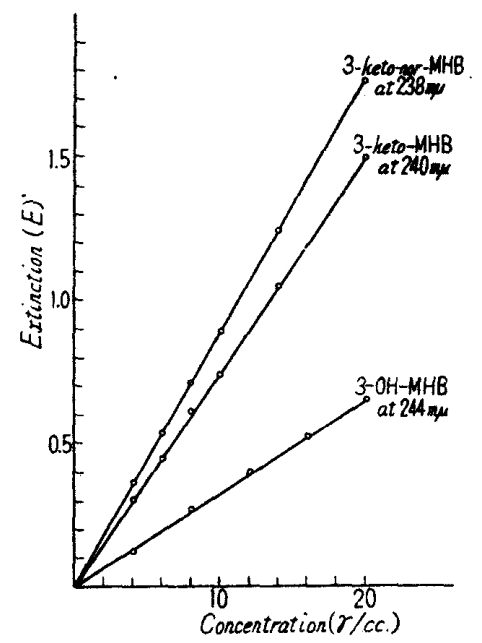

Fig 1. Relation between Extinction at Max Absorption and Concentration of 3-Keto-nor-MHB, 3-Keto-MHB, or 3-OH-MHB

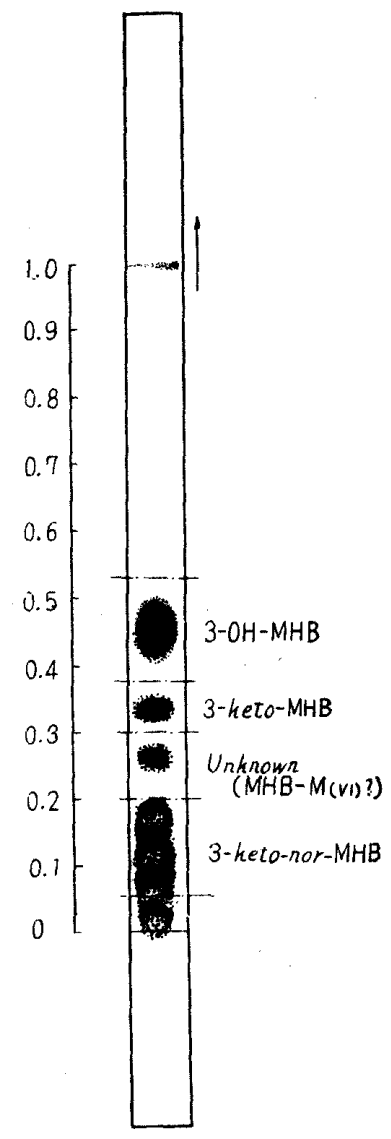

Fig. 2. Chromatogram of MHB Metabolites in Urine
Therefore, on the basis of spectrophotometric data, the concentration of the metabolites can easily be determined.

MHB was given orally by stomach tube to male rabbits after fasting for $24 \mathrm{hrs}$. as freshly prepared aq. solution. containing 1.1 equiv. $\mathrm{NaOH}$ in a dose of 100,200 , and 300 $\mathrm{mg} . / \mathrm{kg}$. body weight. These doses were given to three rabbits alternately, every 7 days. The urine was collected by catheter $24 \mathrm{hrs}$. later, and the urine excreted already in the cage was collected into a bottle. These were combined and filtered through a cotton.

Determination of metabolite in urine-In a glass-stoppered tube were placed $1.0 \mathrm{cc}$. of urine, $0.5 \mathrm{cc}$. of $\mathrm{H}_{2} \mathrm{SO}_{4}(0.85 \sim$ $6.0 \%$; adjusting $\mathrm{pH}$ of medium to 3.0 ), and $12.5 \mathrm{cc}$. of AcOEt. After shaking the mixture for 10 mins., $10 \mathrm{cc}$. of the AcOEt phase was pipetted out and evaporated to drynese. The residue was dissolved in $0.5 \sim 1.0 \mathrm{cc}$. of $\mathrm{MeOH}$ and a portion. of this solution was applied on a filter paper. A quantity of $20 \sim 50 \gamma$ of barbiturates in a volume of $0.05 \sim 0.1 \mathrm{cc}$. was suitable for chromatography. Samples were subjected to ascending chromatography for $16 \sim 18 \mathrm{hrs}$. on Toyo Roshi No. 50 filter paper $(2 \times 40 \mathrm{~cm}$.) which was previously treated with borate- $\mathrm{NaOH}$ buffer $(\mathrm{pH} 11)$, using $\mathrm{BuOH}$ saturated with borate- $\mathrm{NaOH}(\mathrm{pH}$ 11) as the solvent. After development the strips were removed and air-dried. One of them was sprayed with $1 \%$ aq. solution of $\mathrm{NaIO}_{4}$ and $1 \% \mathrm{KMnO}_{4}$ solution, then the other strips were cut into sections at approximate $\mathrm{Rf}$ values corresponding to each spot (Fig. 2). In all, three sections each with $\operatorname{Rf} 0.05 \sim 0.20,0.30 \sim 0.375$, and $0.375 \sim 0.525$ were cut out for 3-keto-nor-MHB, 3-keto-MHB, and 3-OH-MHB, respectively. These sections were eluted with $5.0 \mathrm{cc}$. of borate- $\mathrm{NaOH}$ buffer $(\mathrm{pH} 11)$ and the extinction was measured at each wave length of maximum optical density; 3-keto-nor-MHB at $238 \mathrm{m \mu}$, 3-keto-MHB at $240 \mathrm{m \mu}$, and 3-OH-MHB at $244 \mathrm{m \mu}$. For the blank, 24-hr. urine collected just before the administration of MHB was examined in the same manner as the sample. 


\section{Results and Discussion}

When known amounts of a mixture of authentic 3-keto-nor-MHB, 3-keto-MHB, and $3-\mathrm{OH}-\mathrm{MHB}$ were added to the urine of rabbits, extracted, and estimated according to the method described, recovery rate was $87 \%$ for $3-$ keto-nor-MHB and 3-OH-MHB and $85 \%$ for $3-$ keto-MHB.

Bush, et $\left.a l .{ }^{6}\right)$ reported that after administration of MHB to dogs, only a trace of unchanged material and of nor-MHB, a small amount of keto-MHB I and II, and 5 $6 \%$ of 3-keto-nor-MHB were isolated in the urine.

Previously, ${ }^{4,5)}$ we isolated six metabolites from the urine of rabbits receiving MHB. The yield of these products as pure crystals was $0.2 \sim 0.5 \%$ (3-keto-nor-MHB), $1.0 \sim$ $2.0 \%$ (3- keto-MHB), $\quad 4.0 \sim 5.0 \%(\alpha-3-\mathrm{OH}-\mathrm{MHB}), \quad 1.9 \sim 2.5 \%(\beta-3-\mathrm{OH}-\mathrm{MHB}), \quad 0.2 \sim 0.3 \%$ (ureide), and $0.3 \sim 0.5 \%(\mathrm{MHB}-\mathrm{M}(\mathrm{VI})$ )(total $8.9 \sim 9.5 \%)$.

Now, as shown in Table I, the present method gave a more precise results concerning the yield of the three main metabolites. From these results it was clear that the major product is 3-OH-MHB, and that 3-keto-nor-MHB and 3-keto-MHB are excreted almost equally. There was scarcely any marked difference between respective yield and dosage, and as for the total quantity of excreted metabolites, the difference depending on dosage was not significant.

A small quantities of ureide and MHB-M (VI) would be added to the total yield described above but the fate of remainder was undetectable.

A number of ultraviolet spectrophotometric procedures ${ }^{7)}$ have been developed for the determination of barbiturates in the biological fluids and organs, but those were difficult to simultaneously determine the excreted compounds which contained barbituric acid ring.

TABLE I. Yield of 3-Keto-nor-MHB, 3-Keto-MHB, and 3-OH-MHB in the 24-hr. Urine of Rabbits Administered MHB

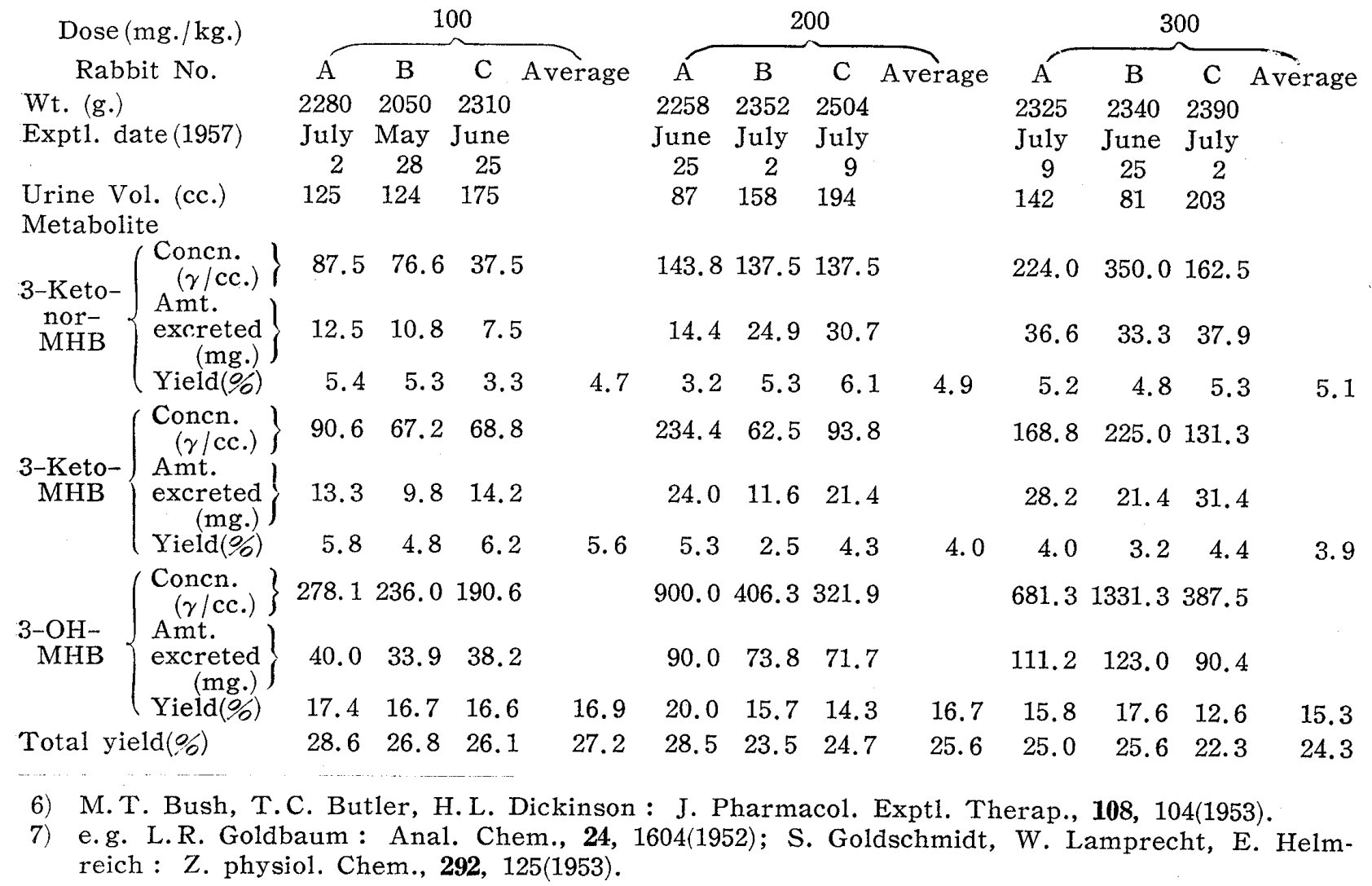


The present procedure based on separation of urinary products by paper chromatography, elution of each section, and estimation by ultraviolet spectrophotometry is able to simultaneously determine the three metabolites in the urine of rabbits receiving MHB.

Bush, et $a l^{6}$. suggested that keto-nor-MHB is produced by the demethylation of previously oxidized MHB. Although from our experiment it was clear that the amount of the hydroxylation product of MHB is predominant, the pathway and the mechanism of degradation of MHB will be clarified by further studies on the interconvertsion of these metabolites, and for these purpose, our quantitative method will be expedient.

\section{Summary}

Method of simultaneous determination of three main metabolites of MHB in urine by paper chromatography in conjunction with ultraviolet spectrophotometry was described.

Approximately $25.7 \%$ of $\mathrm{MHB}$ administered was converted to 3-keto-nor-MHB (4.9\%), 3-keto-MHB (4.5\%), and 3-OH-MHB (16.3\%), and excreted in amounts independent: of the dosage administered.

(Received September 2, 1957) 